Abstracts: Session III

stitutively operational in cultured cells. However, p53 does not regulate expression of $M d m 2$ constitutively in adult murine tissues. Instead p53 requires a stimulus, such as whole-body ionizing irradiation, to stimulate $M d m 2$ expression. These results indicate that p53 regulates $M d m 2$ expression only under certain conditions of stress. We propose that MDM2 regulates p53 only under conditions in which p53 regulates $M d m 2$. To test our hypothesis, we have generated mice carrying a conditionally null allele of $M d m 2$. We have excised exons 7-9 in the germ line and shown that the conditional allele functions as a wild-type allele before excision and as a null allele after excision. We will report whether loss of $M d m 2$ in adult tissues increases p53 levels and activities constitutively or only following a stressor, such as whole-body ionizing irradiation. This study has clinical relevance since the interaction of $\mathrm{p} 53$ and MDM2 has become a target for new anticancer agents.

Peters, Mette A.

\section{Genome-wide scan for high-risk prostate cancer families with breast cancer reveals new loci for prostate cancer and breast cancer}

Mette A. Peters ${ }^{1}$, Janet L. Stanford ${ }^{1}$, Mike D. Badzioch², Suzanne Kolb ${ }^{1}$, Marta Janer ${ }^{3}$, Ellen L. Goode ${ }^{2}$, Mark Gibbs ${ }^{1}$, Leroy Hood ${ }^{3}$, Elaine A Ostrander ${ }^{1} \&$ Gail P. Jarvik ${ }^{2}$

${ }^{1}$ Fred Hutchinson Cancer Research Center, Seattle, Washington, USA

${ }^{2}$ University of Washington, Seattle, Washington, USA

${ }^{3}$ Institute for Systems Biology, Seattle, Washington, USA

Hereditary prostate cancer is a complex disease, and in some families it seems to segregate with other cancers. Epidemiological and genetic studies have reported familial clustering of prostate and breast cancers. As part of a genome-wide search for prostate cancer genes, we performed linkage analyses in 27 families with three or more affected men with prostate cancer who had at least one first-degree relative with breast cancer. To maximize homogeneity, we stratified these families by the pattern of breast cancer (one case, $n=14$; two cases, $n=6$; any ovarian cancer, $n=7)$. Families were stratified into early- (66 yr) or later-onset (= $66 \mathrm{yr}$ ) prostate cancer on the basis of median age at diagnosis. GENEHUNTER was used to compute multipoint NPL scores for prostate cancer linkage using 380 genomic scan markers. Stratified analyses revealed three chromosomal regions with NPL scores of 3.0: (1) chromosome 7q, NPL=3.98 ( $P=0.002)$ at markers D7S1826-D7S1805 in later-onset prostate cancer families with breast and ovarian cancer; (2) chromosome 14q, NPL=3.47 $(P=0.01)$ at marker D14S587 in early-onset prostate cancer families with more than two first-degree relatives with breast cancer and (3) chromosome Xq, NPL $=3.12(P=0.003)$ at marker GATA172D05 in later-onset prostate cancer families with only one relative with breast cancer. None of these families had evidence of significance linkage on chromosomes 13 or 17 . These data supply further evidence for prostate cancer susceptibility genes on chromosomes 14 and $\mathrm{X}$, and they highlight a new region of interest on chromosome 7 that may be involved in the etiology of both prostate cancer and breast cancer.
Petros, John

[5]

\section{Classification of adult renal epithelial neoplasms by gene expression profiling using CDNA microarrays}

Petros, John 1,2,3, Andrew Young ${ }^{1}$, Mahul Amin ${ }^{1}$, So-Dug Lim¹, Fray Marshall ${ }^{1,2}$, James Madara ${ }^{1} \&$ Andrew Neish ${ }^{1}$

${ }^{1}$ Departments of Urology and Pathology, Emory University, Atlanta, Georgia, USA ${ }^{2}$ Winship Cancer Institute, Atlanta, Georgia, USA

${ }^{3}$ Atlanta Veterans Administration Medical Center, Atlanta, Georgia, USA

We analyzed expression of 7,075 genes in four conventional renal cell carcinomas (RCCs), one chromophobe RCC, and two oncocytomas using complementary DNA microarrays. Expression profiles were compared between tumors using hierarchical clustering algorithms. Tumors segregated into two major gene expression classes correlated with histopathological diagnoses; specifically, conventional RCCs were clearly distinguished from chromophobe RCCs and oncocytomas. We classified tumors with high resolution using a selected panel of 70 differentially expressed genes. Chromophobe carcinoma and oncocytomas overexpressed strikingly similar genes, including several related to oxidative phosphorylation and several expressed normally by the distal nephron; these findings are consistent with the mitochondrion-rich morphology of these tumors and the theory that both lesions are related histogenetically to distal nephron epithelium. Conventional RCCs underexpressed mitochondrial and distal nephron genes, and they were further distinguished from chromophobe RCCs and oncocytomas by overexpression of vimentin and molecules related to class II of the major histocompatibility complex. Expression patterns of four genes (those coding for vimentin, CD74, parvalbumin, and galectin-3) were validated in 34 additional tumors by immunohistochemistry. Vimentin was a sensitive, specific marker for conventional RCCs, and parvalbumin was a promising marker for chromophobe RCCs and oncocytomas. Gene expression profiling identified distinct expression patterns related to the pathobiology of renal neoplasms and was an effective approach for discovering immunomarkers for renal tumor subtypes.

Plaschke, Jens [6]

\section{Near-complete association betw een microsatellite instability and abnormal immunostaining of the mismatch repair proteins hMSH2, hMSH6, hMLH1 and hPMS2 and involvement of hMSH6 in sporadic and hereditary colorectal cancers}

Jens Plaschke ${ }^{1}$, Stefan Krüger ${ }^{1}$, Stephan Haas $^{1}$, Steffen Pistorius ${ }^{2}$, Hans-D. Saeger ${ }^{2} \&$ Hans K. Schackert ${ }^{1}$

${ }^{1}$ Department of Surgical Research, Technical University of Dresden, Dresden, Germany

${ }^{2}$ Department of Visceral, Thoracic and Vascular Surgery, Technical University of Dresden, Dresden, Germany

Germline mutations in human mismatch repair genes (mostly in hMSH2 and hMLH1, and recently also in hMSH6) have been found to be associated with the hereditary nonpolyposis colorectal cancer (HNPCC) syndrome. HNPCC tumors are phenotypically characterized by a high level of microsatellite instability (MSI$\mathrm{H})$. Moreover, about $10-15 \%$ of sporadic colorectal cancers are MSI-H, predominantly on the basis of epigenetic silencing of hMLH1 by promoter methylation, 